\title{
Distribución de la propiedad rural en Colombia en el siglo XXI
}

\author{
Distribution of rural property in Colombia during the XXI Century \\ Fabio Alberto Pachón Ariza' ${ }^{1}$ (D) \\ 'Facultad de Ciencias Agrarias, Grupo de Investigación Estudios Rurales y Soberanía Alimentaria (ERSoA), Universidad Nacional de \\ Colombia (UNAL), Bogotá, Colombia. E-mail: fapachona@unal.edu.co
}

\begin{abstract}
Cómo citar: Pachón, F. A. (2021). Distribución de la propiedad rural en Colombia en el siglo XXI. Revista de Economia e Sociologia Rural, 60(4), e242402. https://doi.org/10.1590/1806-9479.2021.242402
\end{abstract}

\begin{abstract}
Resumen: La concentración de la tierra en Colombia es un problema histórico aún sin resolver; situación que también es común en otros países del continente. América Latina y especialmente Sur América, muestran un coeficiente de Gini de tierras alto en comparación con otros continentes. Paraguay y Chile son los países que mayor concentración de tierra alcanzan en Sur América; mientras que Colombia, además de la alta concentración, sufre de problemas asociados con un conflicto armado estrechamente ligado al problema agrario. Esta concentración evidencia el fracaso que ha tenido la política de tierra implementada durante el Siglo XX, tanto la liderada por el Estado, como aquella guiada por el mercado. Este artículo muestra con base en cifras oficiales la evolución de la distribución de la tierra durante las dos primeras décadas del Siglo XXI, evidenciando la persistencia de una histórica concentración y los escasos resultados de la implementación de los Acuerdos de Paz en cuanto a su distribución. Igualmente propone los aspectos que deberían ser tenidos en cuenta para llevar a cabo una reforma agraria alternativa a las visiones tradicionales, una política de tierras basada en la comunidad.
\end{abstract}

Palabras clave: coeficiente de Gini, concentración de tierras, desigualdad, política de tierras.

\begin{abstract}
Land concentration in Colombia is a historical problem still unsolved; a situation that is also common in other countries of the continent. Latin America and especially South America show a high Gini coefficient for land than other continents. Paraguay and Chile are the countries with the highest land concentration in South America; while Colombia, in addition to the high concentration, suffers from problems associated with an armed conflict closely linked to the agrarian problem. This concentration shows the failure of the land policy implemented during the $20^{\text {th }}$ century, both that led by the State and that guided by the market. This article shows, based on official data, the evolution of land distribution during the first two decades of the XXI century, evidencing the persistence of a historical concentration and the limited results of the implementation of the Peace Agreement in terms of its distribution. It also proposes the aspects that should be taken into account to carry out an alternative agrarian reform to traditional views, a communitybased land policy.
\end{abstract}

Keywords: Gini coefficient, land grabbing, inequality, land policies.

\section{Introducción}

La distribución de la propiedad rural en Colombia es un problema central aún no resuelto. Esta situación se ha convertido en uno de los mayores determinantes del conflicto armado en el país (Berry, 2017; Brittain, 2005; Karl, 2017; Nilsson \& Taylor, 2017). A pesar de que la concentración de la propiedad rural no es un problema exclusivo de Colombia, la violencia con que este fenómeno se ha consolidado, sí ha sido muy particular en el país, por lo menos en comparación con lo evidenciado en el continente (Borras, 2003; LeGrand et al., 2017; Petras \& Veltmeyer, 2002; Souza \& Silva, 2011). América Latina muestra cifras que contundentemente evidencian la situación de la distribución de la tierra que, a juicio de Gómez et al., (2015) estaría relacionado con una acumulación primitiva; remontándose a la época colonial, en la que la corona española, mediante la Mita y la Encomienda fortaleció la constitución de lo que en el 
futuro serían las Haciendas (Quintero, 1988). Desde ese momento y hasta nuestros días, los hacendados han ejercido control en diferentes escenarios, especialmente en lo político (Machado \& Vivas, 2009), con el fin de garantizar los privilegios de los que han gozado.

\subsection{Reforma Agraria Contemporánea}

Las políticas de reforma agraria están estrechamente relacionadas con la concentración de la tierra, y existen dos grandes vertientes bien diferenciadas en cuanto a quién debería orientar estas políticas. Por un lado, está aquella que ubica al Estado como el líder y guía de este proceso; en la otra orilla, otra proclive a que sea el mercado el que se encargue de ajustar la distribución (Borras, 2003). El ala proclive al mercado critica la visión estatal, en la medida en que existe la predisposición a pensar que este tendería a limitar la propiedad permitiendo un máximo de tamaño de predios; también se argumenta que para el Estado es sumamente costoso garantizar la limitación a la propiedad, lo cual genera corrupción e inseguridad jurídica al propietario. Igualmente se plantea que implementar políticas de expropiación a la gran propiedad en favor de campesinos lleva a ineficiencias económicas en la medida en que se generan unidades más pequeñas y menos productivas; costos asociados a créditos y asistencia estatal a los pequeños propietarios; distorsión en el mercado de tierras, promoviendo informalidad en las transacciones y evitando la titulación; e inflación en los precios (Deinlnger \& Binswanger, 1999).

El ala proclive al Estado plantea una reforma agraria cuya acción se basa en los beneficios que una equitativa distribución de la propiedad rural genera en cuanto a la producción agropecuaria, distribución de ingresos, generación de empleo, disminución de la pobreza, relaciones más equitativas entre hombres y mujeres (Aliber \& Cousins, 2013; Kay, 1998), así como de lo rural con lo urbano.

Sin embargo, estas dos vertientes dejan de lado aspectos de gran importancia que en la actualidad deben ser discutidos: el significado que puede tener la tierra para una comunidad; nociones plurales de propiedad; así como la diversidad de contextos políticos y económicos en los que se implementa la política. En este escenario, una tercera vertiente toma fuerza, dando un giro hacia una política de tierra guiada por la comunidad, con una orientación "de abajo hacia arriba", que le otorga al Estado una legitimidad para conectarse con las bases y entender sus necesidades y, de esta manera, explorar instituciones y prácticas más relacionadas con lo local (Sikor \& Müller, 2009).

\subsection{Cifras de concentración de tierra}

Una de las formas más comúnmente usadas para medir la desigualdad en la distribución de la tierra es el Coeficiente de Gini, el cual es una medida de dispersión de una distribución que oscila entre 0 y 1, en la que 1 corresponde a una concentración total (Hoffmann, 1979). En América Latina el Coeficiente de Gini para la tierra es de 0,79; mientras que en Europa es de 0,57; en África 0,56 y en Asia 0,55 (Bautista et al., 2017; Guereña \& Burgos, 2016). Estas cifras se muestran detalladas en la Tabla 1 y Tabla 2, lo que permite apreciar que la concentración de la propiedad rural es un problema histórico, sin voluntad política para ser resuelto, pero cada vez más importante en el escenario económico, político y alimentario, debido a que, desde diferentes perspectivas, una gran variedad de actores indaga sobre las consecuencias que dicha desigualdad en la distribución de la tierra tiene para la sociedad en general (Berry, 2017; Nilsson \& Taylor, 2017). 
Tabla 1. Coeficiente de Gini de tierras de 16 Países de América Latina y el Caribe, 1970-1994

\begin{tabular}{|c|c|c|c|}
\hline \multirow{2}{*}{ País } & \multicolumn{3}{|c|}{ Coeficiente de Gini } \\
\hline & 1970 & 1985 & 1994 \\
\hline Chile & 0,92 & --- & 0,92 \\
\hline México & 0,93 & --- & --- \\
\hline Paraguay & --- & 0,93 & 0,93 \\
\hline Brasil & 0,84 & 0,85 & 0,81 \\
\hline Colombia & 0,86 & 0,79 & 0,79 \\
\hline Costa Rica & 0,81 & 0,80 & --- \\
\hline Ecuador & 0,81 & --- & --- \\
\hline El Salvador & 0,80 & 0,83 & --- \\
\hline Panamá & 0,77 & --- & 0,85 \\
\hline Perú & 0,88 & 0,83 & 0,86 \\
\hline Venezuela & 0,90 & 0,89 & --- \\
\hline Honduras & 0,71 & --- & 0,66 \\
\hline Jamaica & 0,79 & --- & --- \\
\hline Puerto Rico & 0,76 & 0,77 & --- \\
\hline República Dominicana & 0,78 & 0,73 & --- \\
\hline Uruguay & 0,81 & 0,80 & 0,76 \\
\hline
\end{tabular}

Fuente: (Comisión Económica para América Latina, 2004)

Tabla 2. Coeficiente de Gini de tierras en América Latina

\begin{tabular}{lcc}
\multicolumn{1}{c}{ Subregión / País } & $\begin{array}{c}\text { Coeficiente de Gini en } \\
\text { distribución de la tierra }\end{array}$ & Año del Coeficiente \\
\hline América del Sur & 0,93 & $\mathbf{0 , 8 5}$ \\
Paraguay & 0,91 & 2008 \\
Chile & 0,88 & 1997 \\
Colombia & 0,88 & 2009 \\
Venezuela & 0,87 & 1997 \\
Brasil & 0,86 & 2006 \\
Perú & 0,84 & 1994 \\
Uruguay & 0,83 & 2000 \\
Argentina & 0,8 & 1998 \\
Ecuador & 0,77 & 2000 \\
Bolivia & & 1984 \\
América Central & 0,84 & 2003 \\
Guatemala & 0,81 & 2001 \\
El Salvador & 0,77 & 2001 \\
Panamá & 0,72 & 2001 \\
Nicaragua & 0,67 & $\mathbf{0 , 7 5}$ \\
Costa Rica & & \\
\hline
\end{tabular}

Fuente: Guereña \& Burgos (2016, p. 22).

Profundizando en la distribución de la tierra rural en algunos de los países de América Latina se podría afirmar lo siguiente. En primer lugar, la escasez de datos recientes es un factor común en todo el continente; por ejemplo, Bolivia, Venezuela, Perú y Chile disponen de datos de hace más de 20 años. En segundo lugar, América del Sur tiene un coeficiente de Gini más alto con un 0,85, en comparación con América Central que alcanza un Gini de 0,75. 
Igualmente, Paraguay con 0,93 es el país más desigual de la región, mientras que Costa Rica con 0,67, alcanza el menor índice.

Un tercer aspecto a destacar es que Chile, con un coeficiente de Gini por encima de 0,90, se constituye en uno de los casos en los que se configuró una reconcentración de la tierra, donde más de la mitad de los beneficiarios de reforma agraria perdieron sus predios debido a las deudas que se acumularon en el proceso de compra de los mismos y de establecimiento de la producción, siendo en su mayoría finalmente adquirida por grandes productores (Kay, 2002). Esta concentración se manifiesta en que el $98 \%$ de las producciones ocupan el $21 \%$ de la superficie de tierra; y al otro extremo, predios mayores de 2000 hectáreas son el 0,5\% del total de explotaciones y ocupan el 70\% de las tierras (Echeñique, 2011).

Finalmente, en algunos países del Sur del continente, como Paraguay, Brasil, Argentina, Uruguay y Bolivia, la expansión de cultivos con una clara orientación exportadora ha hecho que se concentre cada vez más la propiedad rural. En Argentina, por ejemplo, con un coeficiente de Gini de 0,83 los cultivos de soya ocupan más del $50 \%$ de su superficie arable; $y$, si sumamos cultivos de girasol y maíz, cubren casi el 70\% (Reboratti, 2010). A pesar de que se cuenta desde el 2013 con el Programa Nacional de Titulación y Arraigo Rural (PRONTAR), su alcance ha sido precario, específicamente porque busca sanear situaciones de tenencia de tierra, mas no su distribución (Bautista et al., 2017).

Paraguay por su parte, mostraba en 2002 la más alta concentración de tierra rural en el continente. Según el Censo Agrícola de ese año, el 2,6\% de los propietarios tenía el $85 \%$ del territorio, y la producción estaba concentrada en cultivos de soya y ganadería; en 2002 los cultivos de agroexportación ocupaban 3 millones de hectáreas, en 2014 lo hacían en más de 6 millones; en 2018 la agricultura campesina ocupaba el 6,48\% de las tierras, mientras que los agronegocios el 93,51\% (Palau, 2019). En el país existe el Instituto Nacional para el Desarrollo Rural y de la Tierra (INDERT) y el Instituto Nacional del Indígena para campesinos y poblaciones originarias que se ocupan de los asuntos de tierras. Igualmente se ha implementado el Sistema de Información de Recursos de la Tierra (SIRT), que busca actualizar los registros agrarios, pero que se ha utilizado para legalizar títulos ilegalmente adquiridos, beneficiando a sojeros y ganaderos que usurparon tierras en el marco de la reforma agraria (Bautista et al., 2017).

En Uruguay, según el censo Agropecuario 2014, el número total de predios se redujo en un $20 \%$, especialmente en aquellos menores de 100 ha; y la concentración se ejemplifica en que el 2,5\% de los propietarios ocupan el 34\% de la tierra (Uruguay, 2015). Los cultivos de cereales ascendieron a 1,6 millones de ha en 2013; la soya ocupó 1,3 millones en el mismo año, la producción campesina disminuyó en 52\% entre 2004 y 2014; 12 empresas con participación de capital extranjero explotaban el 35\% de la producción agrícola en 2009 (Favat \& Gutiérrez, 2010).

Brasil muestra una tendencia a la concentración, que no ha sido alterada en los últimos censos agropecuarios (1996 y 2006), lo que se refleja en un coeficiente de Gini de 0,87; el $36 \%$ de la superficie de tierra del país se consideraba como tierras 'griladas', es decir, que sus poseedores no pueden comprobar la legalidad de su propiedad, demostrando la falta de control público sobre las tierras (Bautista et al., 2017). Los cultivos de soya en Brasil pasaron de ocupar 1,5 millones de ha en 1970 al 45\% del área agrícola del país en 2014, produciendo más de 60 millones de toneladas al año que representan el 38\% de las exportaciones mundiales de este producto; los cultivos de caña de azúcar para etanol ocupaban más de 55 millones de ha en 2013 (Gras \& Göbel, 2014).

En Bolivia, en donde no hay datos recientes de distribución de la propiedad rural, la producción de soya pasó de 234 mil toneladas en 1990 a 2,4 millones en 2012, dominado en un $90 \%$ por grupos agroindustriales transnacionales que se han adueñado de las mejores tierras 
(Eguren, 2018). El gobierno boliviano en 2006 cambió el enfoque de la política mediante la Ley de Reconducción Comunitaria de la Reforma Agraria, buscando sanear y distribuir la tierra, promoviendo la tenencia y generando condiciones materiales para el acceso; en 2016 había saneado el $77 \%$ de las propiedades, titulando 55 millones de hectáreas, de las cuales 21 millones habían sido entregadas a 1,5 millones de campesinos; 24 millones a 372 mil indígenas y 9 millones a 9.700 agroindustriales (Bautista et al., 2017).

Dado este contexto, el objetivo de este artículo es analizar la distribución de la propiedad rural en Colombia durante las dos primeras décadas del Siglo XXI, especialmente debido a que, desde 2016, el país inició un proceso de Reforma Rural Integral en el marco de los acuerdos de Paz entre el gobierno y las FARC-EP.

\section{Materiales y métodos}

La información base para hacer el análisis de la distribución de la tierra en Colombia durante las dos primeras décadas del Siglo XXI fue obtenida de las 'Estadísticas Catastrales de Distribución Rural por Rangos de Superficie' del Instituto Geográfico Agustín Codazzi (IGAC). Los años tenidos en cuenta para el análisis fueron 2004, 2010, 2013, 2014, 2018 y 2019. Dichas estadísticas muestran diferentes rangos de superficie de tierra, que van desde predios menores de 1 hectárea, hasta predios mayores de 2000 ha. Para cada uno de los rangos se identifica el número de predios y el número de propietarios. Los datos analizados no incluyen el departamento de Antioquia debido a que su información catastral está descentralizada en el catastro departamental.

Con base en esta información se utilizó la fórmula propuesta en el estudio titulado 'Atlas de Distribución de la Propiedad Rural en Colombia', investigación liderada por el Instituto Geográfico Agustín Codazzi (2012), en conjunto con la Universidad de los Andes de Bogotá y la Universidad de Antioquia, en la que se determinó el Coeficiente de Gini de tierras para el año 2012. La fórmula utilizada fue la siguiente:

Gini $=1+\frac{1}{n}-\frac{2}{n^{2} y} \sum_{i}^{n} y i(n+1-i)$

donde:

$n=$ Total de la población

$y=$ Promedio del área de terreno medido en hectáreas

$y i=$ Área del terreno del predio $i$

\section{Resultados}

La Tabla 3 muestra los resultados organizados en 13 diferentes rangos de tenencia, desde predios menores de 1 hectárea, hasta mayores de 2000 ha; para cada uno de los rangos definidos se muestra el número de propietarios y la superficie que ellos ocupan en hectáreas.

Tomando como base los resultados de la Tabla 3 se calculó el porcentaje de propietarios y el porcentaje de tierra que ocupan, organizado por rangos de tenencia. La Figura 1 muestra los resultados porcentuales para el año 2004, mientras que la Figura 2 hace lo propio para el año 2019. En estas figuras se evidencia la evolución de la concentración de la tierra, en la que los extremos de propiedad, es decir predios muy grandes o muy pequeños, están en manos de los extremos contrarios de propietarios. En otras palabras, muy pocos propietarios poseen los predios muy grandes, quienes acumulan más del $97 \%$ de la superficie de tierra rural. Por el 


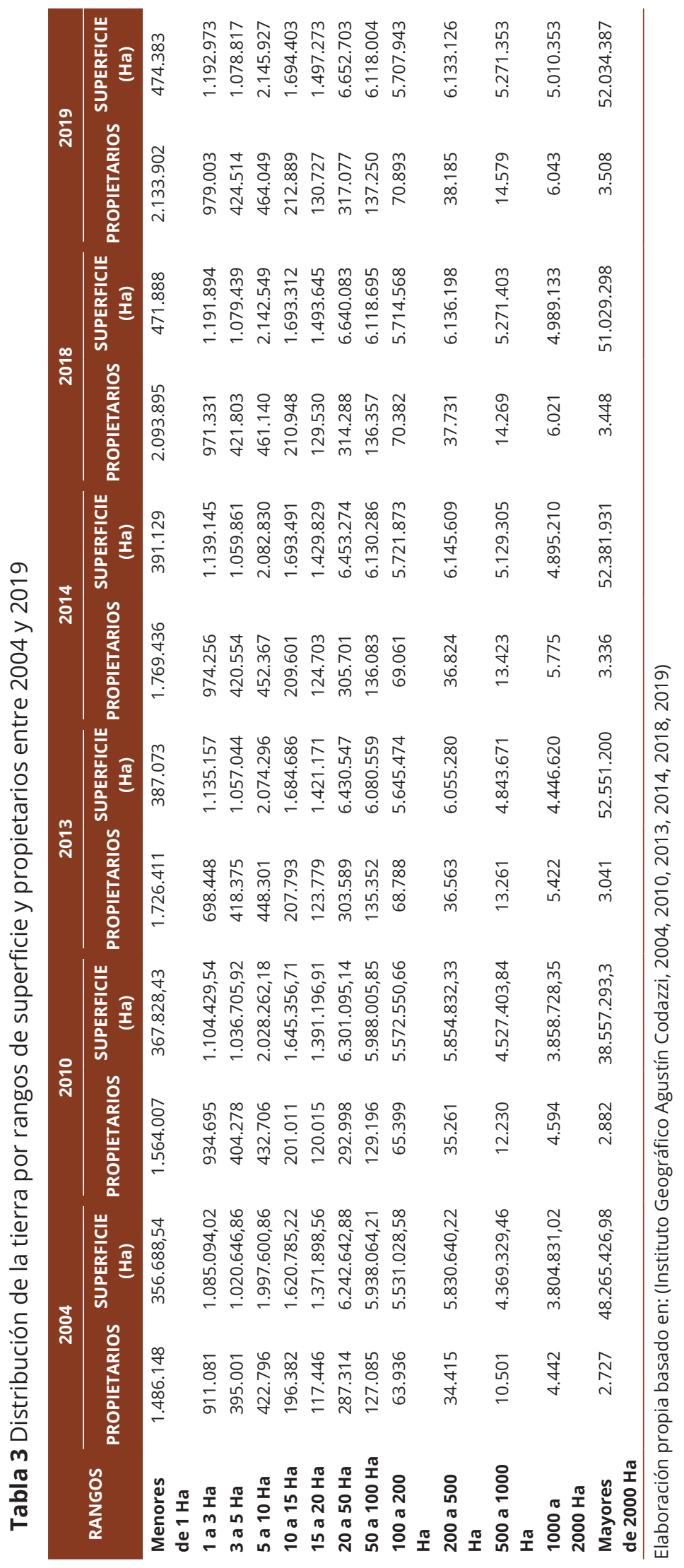


contrario, muchos propietarios, más del $97 \%$, poseen predios muy pequeños, los cuales suman menos del $3 \%$ de la superficie de tierra rural.

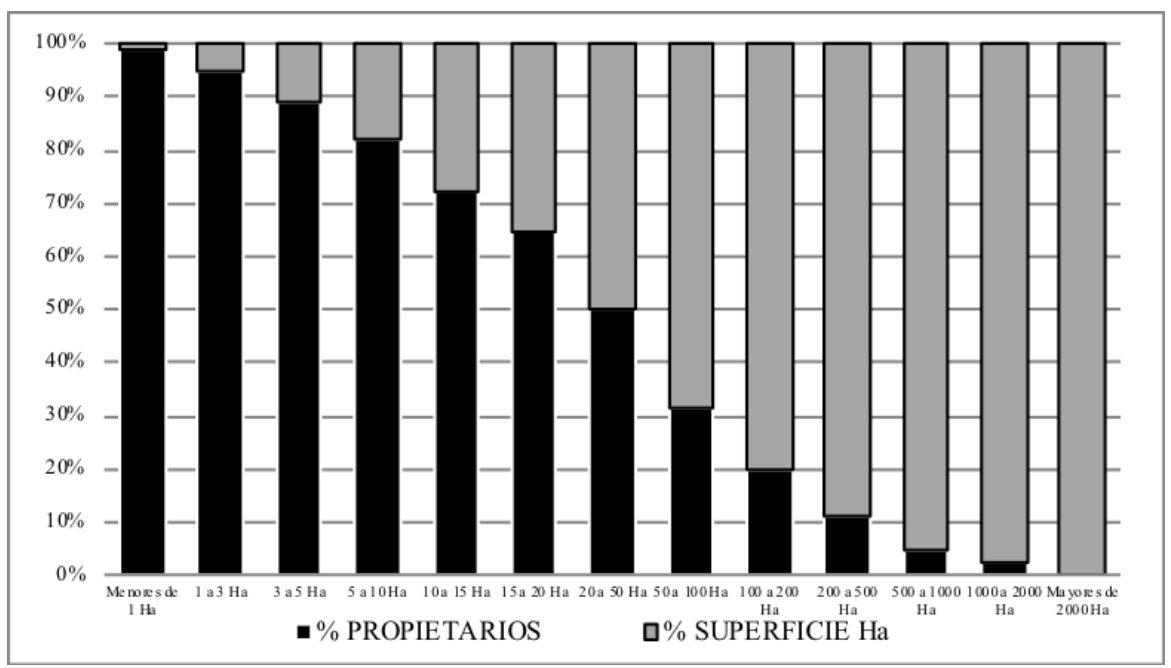

Figura 1 Porcentaje de tenencia por tamaño de predio 2004. Elaboración propia basado en: (Instituto Geográfico Agustín Codazzi, 2004)

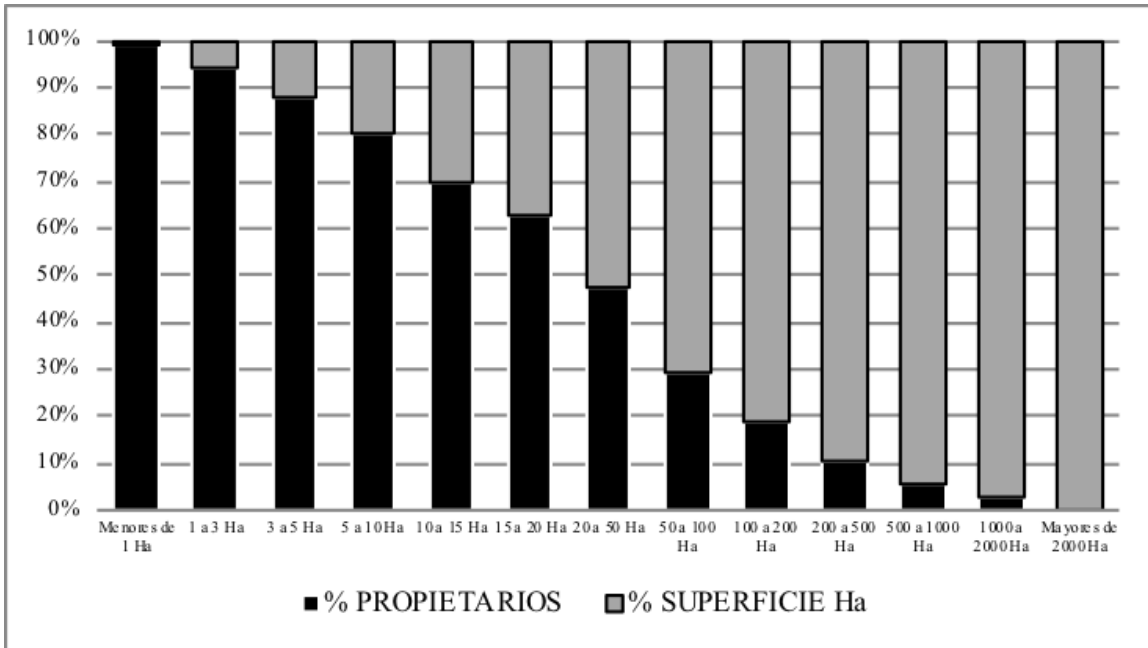

Figura 2 Porcentaje de tenencia por tamaño de predio 2019. Elaboración propia basado en: (Instituto Geográfico Agustín Codazzi, 2019)

Como se evidencia en estos resultados, la situación de la concentración de la tierra no cambia a lo largo de las dos primeras décadas del Siglo XXI; los extremos continúan casi inamovibles en el tiempo, como se explicó anteriormente. Sin embargo, en los rangos intermedios de distribución de la propiedad se evidencia que existe una leve tendencia hacia una mayor concentración, lo cual es muy importante dado que allí es donde el mercado de tierras usualmente funciona con base en la oferta y la demanda. A su vez, implementar políticas que desincentivan la concentración en predios muy grandes es poco probable debido a que en muchos casos este fenómeno ha sido mediado por el uso de la fuerza, además que en Colombia la legislación tributaria no permite, vía impuestos, desincentivar la tenencia y el uso inadecuado de las tierras. 
Adicionalmente, los procesos de extinción de dominio son complejos y colmados de trámites en los que la institucionalidad existente con muchas dificultades puede avanzar. En el otro extremo de la balanza: los microfundios, predios muy pequeños con menos de 1 hectárea, no pueden dividirse más; porque llevaría a una mayor precarización de la vida de los campesinos y propietarios rurales de pequeña extensión.

En los segmentos intermedios de distribución de la propiedad, es decir predios de hasta 20 hectáreas, se evidencia la tendencia descrita: muchos propietarios poseen muy poca tierra. En predios superiores a las 50 hectáreas se evidencia que la proporción de tierra aumenta y el número de propietarios cada vez es menor. En el rango de predios entre 20 y 50 hectáreas se encuentra una tendencia hacia la equidistribución: el 50\% de los propietarios ocupa el 50\% de la tierra de ese rango. Como se identifica, estas tendencias se mantienen casi inamovibles en los años de estudio.

La Tabla 4 muestra el coeficiente de Gini de tierra en Colombia durante las dos primeras décadas del Siglo XXI, el cual permanece constante. No obstante, aumenta en comparación con las cifras del siglo pasado.

Tabla 4. Coeficiente de Gini de tierra años 2004 a 2019

\begin{tabular}{cc} 
AÑO DE ESTUDIO & COEFICIENTE DE GINI \\
2004 & 0,89809 \\
2010 & 0,89207 \\
2013 & 0,89886 \\
2014 & 0,89616 \\
2018 & 0,8952 \\
2019 & 0,8955 \\
\hline
\end{tabular}

Elaboración propia basado en: (Instituto Geográfico Agustín Codazzi, 2004, 2010, 2013, 2014, 2018, 2019)

Gráficamente el Coeficiente de Gini de tierra para el año 2004 y 2019 se muestra en la Figura 3. La línea transversal representa una distribución completamente equitativa o equidistribución: en la que el uno por ciento de la tierra está distribuido entre el uno por ciento de los propietarios, el 50 por ciento de la tierra está distribuida entre el 50 por ciento de los propietarios, y así sucesivamente. El Coeficiente de Gini muestra la distancia entre la distribución real, representada por la curva de Lorenz y la equidistribución (Instituto Geográfico Agustín Codazzi, 2012:13-14).

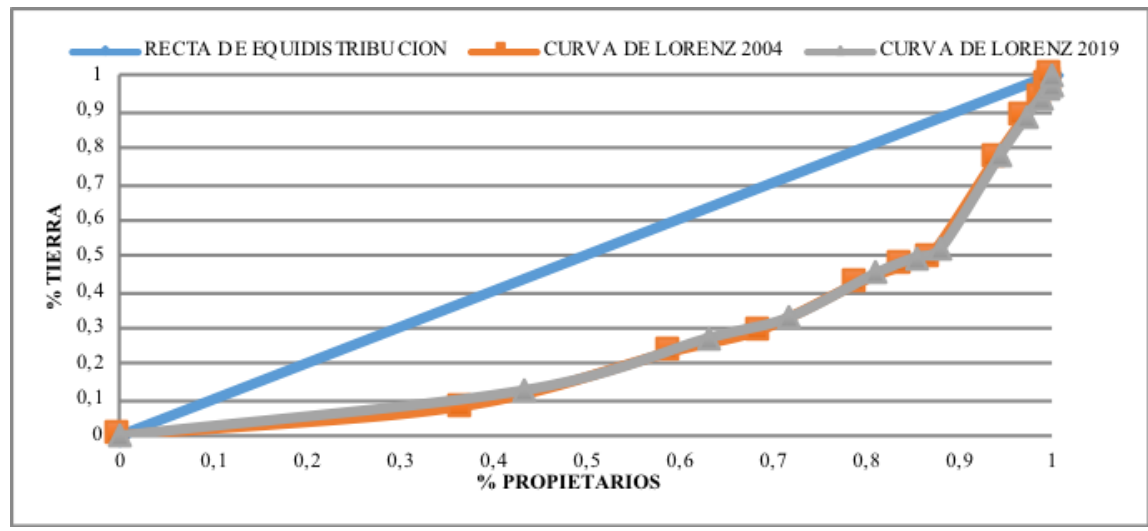

Figura 3. Coeficiente de Gini de tierras años 2004 y 2019 
Como se evidencia, la distancia en la recta de equidistribución y la Curva de Lorenz de los años 2004 y 2019 son casi idénticas. Esto significa que la distribución de la tierra en Colombia durante las dos primeras décadas del Siglo XXI permanece inalterada, y los efectos de las políticas que se han empezado a implementar como resultado del Acuerdo de Paz con las FARC no han logrado disminuir la histórica concentración de la propiedad rural en el país.

Los datos del Coeficiente de Gini demuestran la complejidad del problema de la distribución de la tierra en Colombia; como ejemplo de esto para el año 2004, el 36\% del total de los propietarios ocupaban predios menores a 1 hectárea y corresponden al 0,40\% del total de la superficie de tierra rural del país. La distribución en este mismo rango cambia en el año 2019, cuando el porcentaje de propietarios aumenta al $43,26 \%$ ocupando el 0,50\% de la superficie de tierra rural de Colombia; es decir, un 7\% más de propietarios pasan a ocupar microfundios, los cuales porcentualmente no aumentan de manera significativa en términos de la cantidad de tierra que ocupan.

En el otro extremo, en predios grandes, en el año 2004 el 0,06\% del total de los propietarios ocupaban predios de más de 2000 hectáreas y corresponden al 55,2\% del total de las tierras rurales del país. Se evidencia una disminución no significativa en el área ocupada en el 2019, en el que el $0,07 \%$ de los propietarios ocupaban el $54,7 \%$ de la superficie.

Con relación a los datos que permiten comparar la distribución de la propiedad rural luego de la firma del Acuerdo de Paz entre las FARC y el Estado colombiano, no hay evidencia de grandes avances en ninguno de los rangos de propiedad, los cuales permanecen con similar distribución durante el tiempo. En el año 2013, previo a los acuerdos específicos del punto 1 sobre Reforma Rural Integral, el 3\% del total de los propietarios ocupaban el 78\% del total de la tierra y lo hacían con predios de más de 100 hectáreas; mientras que el 83,5\% del total de los propietarios ocupaban el $6,7 \%$ del total de la tierra distribuida en predios de menos de 10 hectáreas. En 2019, tres años después de la firma del Acuerdo, el 3\% del total de los propietarios ocupaban el $78 \%$ del total de la tierra rural distribuida en predios de más de 100 hectáreas; y en predios de menos de 10 hectáreas, el 85,5\% del total de los propietarios solo ocupaban el $7 \%$ del total de la tierra.

\section{Discusión}

Los análisis relacionados con la distribución de la propiedad rural en Colombia enmarcados en los estudios de las consecuencias de las políticas de reforma agraria han venido adquiriendo una mayor relevancia, especialmente durante la segunda década del Siglo XXI, debido a los diálogos y al posterior Acuerdo de Paz entre las FARC y el Estado colombiano (Bautista et al., 2017; Machado, 2013). Esto se evidencia en que los escritos relacionados con la concentración de la tierra desde diferentes esferas, tanto académicas, gubernamentales, o de organizaciones independientes, han sido numerosos e importantes (Colombia, 2010; Bautista et al., 2017; Borras et al., 2011; Botia-Carreño, 2019; Brittain, 2005; Duarte et al., 2019; Grajales, 2015; Guereña \& Burgos, 2016; Hristov, 2005; Thomson, 2011).

Dentro de esos estudios vale la pena mencionar el Atlas de la Propiedad Rural en Colombia del Instituto Geográfico Agustín Codazzi (2012) en el cual, de manera sistemática, se analiza la concentración de la propiedad durante la primera década del Siglo XXI, teniendo en cuenta las cifras departamentales y nacionales (Tabla 5). Los datos de esa investigación fueron usados por Machado (Machado, 2009) en el Informe de Desarrollo Humano 'Razones para la Esperanza' para demostrar cómo la concentración de la tierra es un problema aún sin resolver en Colombia. 
Tabla 5. Coeficiente de Gini de tierra Colombia 2000 a 2011

\begin{tabular}{cc|}
\hline AÑO DE ESTUDIO & COEFICIENTE DE GINI \\
2000 & 0,854 \\
2001 & 0,856 \\
2002 & 0,855 \\
2003 & 0,855 \\
2004 & $\mathbf{0 , 8 5 6}$ \\
2005 & 0,855 \\
2006 & 0,855 \\
2007 & 0,856 \\
2008 & 0,860 \\
2009 & 0,859 \\
2010 & $\mathbf{0 , 8 6 3}$ \\
2011 & 0,874 \\
\hline
\end{tabular}

Elaboración propia basado en Instituto Geográfico Agustín Codazzi (2012)

Los años en los que el Atlas de tierras y la presente investigación coinciden en sus análisis son 2004 y 2010, cuyos resultados muestran una diferencia entre los dos estudios. Aunque esa diferencia es no significativa, podría ser explicada en un hecho mencionado anteriormente: la presente investigación no incluye los datos del departamento de Antioquia, ya que allí la información catastral se maneja de manera independiente; sin embargo, en ambos estudios el Coeficiente de Gini demuestra la evidente concentración de la tierra en pocos propietarios, representada en predios en gran tamaño.

Del mismo modo, el estudio de Guereña \& Burgos (2016) ubica el Coeficiente de Gini para Colombia del 2009 en 0,88; adicionalmente se relaciona la concentración de la propiedad rural en el país representada en un pequeño porcentaje de la población propietaria de predios de gran tamaño, especialmente mayores de 500 hectáreas, fenómeno que se ha visto incrementado desde 1970. “En Colombia entre 1970 y 2014, mientras que las fincas de más de 500 hectáreas se expandieron del $41 \%$ al $77 \%$ del área agropecuaria, las de menos de 10 hectáreas se contrajeron del 7\% al 4\% del territorio" (Guereña \& Burgos, 2016).

Por otro lado, el estudio 'La política de reforma agraria y tierras en Colombia. Esbozo de una memoria institucional' del CNMH (Centro Nacional de Memoria Histórica, 2013) menciona como uno de los graves problemas a los que se enfrenta el sector rural Colombiano "...la alta concentración de la tierra, la alta concentración de la propiedad reflejadas en un índice Gini de tierras del 0,87; los conflictos de uso del suelo con una preponderante sobre-explotación en ganadería extensiva y la subutilización del suelo en agricultura; altos niveles de informalidad en los derechos de propiedad rurales; el despojo y abandono de tierras por medios violentos y a través de la violación de normas jurídicas (...) atrasos en la actualización catastral y falta de información sobre la tenencia de la tierra (...) configuración de estructuras agrarias ilegales vinculadas al conflicto, el paso de la lucha por la tierra a la disputa de los territorios, y la violencia contra la población rural" (2013:148). Resulta claro que para abordar el problema agrario colombiano es necesario afrontar la concentración de la tierra con unas políticas adecuadas; en caso contrario, la persistencia del problema continuará. En este contexto es importante mencionar que el Acuerdo de Paz y la Reforma Rural Integral (RRI) abrieron una ventana de esperanza que, a pocos años de iniciar su implementación, se hace más pequeña e inalcanzable.

Además, los aspectos que quedaron plasmados en el Acuerdo de Paz y que iban de la mano con la entrega de 3 millones de hectáreas y la formalización de otros 7 millones a los campesinos, son contradictorios, por lo menos en el papel, con la otra apuesta del gobierno plasmada en 
la propuesta por la cual se crean y se desarrollan las Zonas de Interés de Desarrollo Rural, Económico y Social (ZIDRES) mediante la Ley 1776 de 2016 del Congreso de la República de Colombia.

Las ZIDRES buscan que empresas agroindustriales privadas reciban en comodato, concesión, arrendamiento o cualquier otra modalidad contractual, tierras en zonas de difícil condición y en las cuales el Estado colombiano no ha hecho presencia. Plantea que los proyectos que se vayan a ejecutar en este marco deben cumplir con varios requisitos como su enfoque territorial, esquemas claros de viabilidad jurídica y financiera, planes de apoyo en créditos y asistencia técnica a campesinos, quienes serán socios de los emprendimientos, contando con las garantías de compra de la producción y sistemas que garanticen el acceso a la tierra por medio de créditos, entre otros.

En la práctica, la implementación de las ZIDRES se contrapone a la RRI en la medida en que entrega las tierras a empresas, en lugar de hacerlo a los campesinos, privatiza aún más el sector agrícola y vulnera la Soberanía Alimentaria nacional. En otras palabras, la apuesta del 'Estatuto de Desarrollo Rural' liderada por los gobiernos de Alvaro Uribe Vélez entre 2002 y 2010, se consolida por medio de las ZIDRES, legalizando la acumulación ilegal de baldíos de la nación que habían hecho empresas privadas durante muchos años, contribuyendo a la acumulación de tierras en Colombia.

Según Forero Rueda (2020), el 31 de marzo de 2020 habían ingresado al Fondo de Tierras, recién creado por el Acuerdo de Paz y la RRI, 1'000.404 de hectáreas. Sin embargo, a esa fecha ningún campesino en el país por cuenta del Acuerdo de Paz había recibido predio alguno; a pesar de que el tiempo estipulado para dichas entregas finaliza en 2028, resulta un ejemplo sobre la poca ejecución del Acuerdo, que años después de iniciar su implementación, muestra que el avance sobre este particular es muy precario.

En medio de este oscuro panorama, es importante destacar algunos aspectos clave para ser tenidos en cuenta en una política que pretenda superar el problema de la concentración de la propiedad rural en Colombia. Un principio fundamental es que la tierra, a pesar de estar en manos privadas, se puede considerar como un bien de uso común que debe generar un beneficio para todos, puesto que lo que suceda con ella afectará a la sociedad en general (Ostrom, 2009). Por ejemplo, el impacto se produce cuando un poseedor decida usarla para conservar o producir algo que beneficie al resto de la comunidad tal como alimento, agua u oxígeno o, por lo contrario, su uso genere impactos negativos derivados de contaminación de fuentes de agua. Este planteamiento ha sido ampliamente desarrollado en diversos aspectos desde la publicación originaria 'The Tragedy of the Commons' (Hardin, 1968), para este caso se ejemplifica en que aquellos propietarios impulsados por una visión de maximización económica, por intereses relacionados con consolidación de espacios de poder en los territorios o simplemente por un interés especulativo, se apropian cada vez de más recursos. Esto, en un escenario en el que dicho recurso, la tierra, es limitado tanto en cantidad como en calidad, y los propietarios lo usan libremente de acuerdo con sus intereses particulares. Por esta razón, ni el Estado ni los privados, a través de diferentes instituciones, han logrado que los propietarios hagan una distribución y uso del recurso que permite el beneficio común.

Así las cosas, tomando como base los postulados de Hardin y Ostrom, y las limitaciones en la distribución que se han evidenciado, es que se puede describir algunos de los elementos que podría tener una política de tierras que sea liderada por la comunidad (Sikor \& Müller, 2009), en búsqueda de la consolidación de esa tercera vertiente de política de tierras descrita anteriormente; partiendo de una iniciativa distinta al liderazgo estatal o privado que se han implementado en años anteriores. Un primer elemento es el reconocimiento de la comunidad 
y sus diferentes formas organizativas. Una reforma agraria basada en la comunidad, en primer lugar, empodera y se convierte en un importante escenario de lucha política por la reivindicación de los derechos de la misma comunidad; ejemplos de ello pueden ser las comunidades indígenas o negras, cuyas tradiciones y relaciones con el Estado les ha permitido construir espacios territoriales colectivos, contrario a lo que ha sucedido con el campesinado, que han sido tratados con menos reconocimiento de derechos que favorezcan esa misma titularidad. El campesinado debería tener la posibilidad de acceder a derechos de propiedad en zonas colectivas, con gobiernos locales con autoridad para resolver los problemas locales (Fajardo, 2002). Ejemplos de dichas formas de reconocimiento a derechos de propiedad para comunidades campesinas alrededor del mundo son descritos por Sikor \& Müller (2009), en donde el papel del Estado ha sido crucial en favor de dichos cambios y de la protección de los derechos, incluido el derecho a la vida, en estas comunidades.

Un segundo aspecto crucial de una reforma agraria basada en la comunidad es que es más sencillo encontrar una relación comunitaria con la naturaleza desde las organizaciones de base. Algunas razones para la gestión inadecuada, que las instituciones tradicionales del Estado y el mercado han liderado en lo local, incluyen la exclusión de las mismas comunidades en dicha gestión (Ostrom, 2009), o que obedecen a intereses particulares sin arraigo al territorio (Goebel, 2012). En contraste con lo anterior, las normas en una comunidad para establecer las relaciones con la naturaleza son más fáciles de construir cuando los actores son partícipes de ese proceso de decisión, debido precisamente a la relación que existe con ella y la conciencia del beneficio colectivo que esto puede generar (Cárdenas, 2009). Igualmente, cuando las reglas se rompen, es más fácil reflexionar en un escenario comunitario sobre los diferentes tipos de desigualdades que se pueden generar (Sikor \& Müller, 2009).

El tercer aspecto es la noción que adquiere la propiedad en una reforma agraria basada en la comunidad; dándose por sentado que va más allá de las reflexiones económicas; preocupándose por aspectos relacionados con los beneficiados por la explotación de la tierra, en qué condiciones lo hacen, cómo se establecen relaciones de poder bajo la lógica de la tenencia privada, y cómo esto afecta política y culturalmente a la comunidad. El sentido de propiedad desde una base comunitaria abre el debate sobre los usos cotidianos que los propietarios hacen de la tierra (Von Benda-Beckmann et al., 2006), al igual que sobre la superación de la visión económica de la propiedad, en la que el Estado solo es garante de los derechos sobre la misma y su usufructo, convirtiéndose en un facilitador de la tenencia bajo una idea de propiedad comunitaria asociada a diferentes contextos. Lo anterior, en una lógica de derechos individuales o colectivos. Igualmente, otros tipos de propiedad que se basan en los diferentes significados y usos que puede tener la tierra más allá de lo productivo (Sikor \& Müller, 2009).

En Colombia la propuesta de Zonas de Reserva Campesina consagrada en la Ley 160 de 1994, y luego reglamentada por el Decreto 1777 de 1996, podría considerarse que comparte algunas características semejantes a las descritas en una política de reforma agraria de base comunitaria, en la medida en que reconoce a las comunidades la posibilidad de participar de una manera directa en los procesos de planificación de su territorio basado en sus particularidades sociales, culturales, políticas, económicas y ambientales (Quijano-Mejia \& Linares-García, 2017). Sin embargo, debido a la influencia de los círculos interesados en el mantenimiento del statu quo, el contexto neoliberal en el que se establecen desde la Ley 160 de 1994, y los intereses de la institucionalidad encargada de su fomento, su alcance ha sido precario (Fajardo, 2002). Tal vez por esa misma razón es que se han constituido en diferentes partes del país, Zonas de Reserva Campesina de hecho, que se acercan a la construcción de una reforma agraria basada en las comunidades. 
Otras particularidades para el caso colombiano deben acompañar a una reforma agraria basada en la comunidad para alcanzar una más equitativa distribución del recurso. Un aspecto crucial a tener en cuenta es utilizar adecuadamente el concepto que permite medir la tierra en términos de Unidades Agrícolas Familiares (UAF), como instrumento para definir el área necesaria para que una familia campesina pueda vivir en condiciones dignas, las cuales resultan útiles para evidenciar la real concentración del recurso (Colombia, 2010; Botia-Carreño, 2019). En términos de tenencia, no es lo mismo ser propietario de 2000 hectáreas de tierra en el departamento del Vichada donde una UAF puede ser mayor a 500 hectáreas, que ser dueño de estas 2000 hectáreas en el Altiplano Cundiboyacense donde una UAF puede estar entre 5 y 10 hectáreas.

Sobre la base de una medición en UAF, otro elemento a destacar es una reforma de la política tributaria (Hirschman, 2013) que debería estar basada en el número de UAF que un propietario, sea de carácter individual, familiar o empresarial, posea. Una legislación que busque solucionar el problema debería tener tasas impositivas diferenciales para los poseedores de más de 5 Unidades Agrícolas Familiares, quienes deberían pagar altos impuestos por cada Unidad adicional que posea. Esta medida, en teoría, desincentivaría una alta concentración y disminuiría la desigualdad en la distribución, en la medida en que el beneficio que un propietario pueda recibir por cada UAF adicional que posea sea menor a la tasa impositiva que tendría que pagar por tener dicha UAF.

Sumado a lo anterior, un aspecto adicional habría de estar relacionado con que los impuestos a pagar deberían tener en cuenta el uso que se haga de la tierra. Solo para el caso productivo, un propietario que use la tierra en actividades diferentes a la que la vocación de uso que esta le permita, debería pagar impuestos por el uso inadecuado. El uso adecuado debería incluir aquellas visiones que superan lo productivo y que fueron descritas párrafos atrás, concretamente aquellas que han sido construidas con base comunitaria. Sin embargo, un propietario que produzca sus tierras de acuerdo con la vocación de uso, podría ser beneficiario de una rebaja en la tasa impositiva como incentivo para promover una adecuada explotación. Para ejemplificar este punto, sería sujeto de impuestos un propietario que tenga explotaciones ganaderas cuando la vocación de uso de su tierra sea agrícola, o un propietario que tenga explotaciones agrícolas en zonas de conservación.

De la mano con lo descrito, la legislación debería también tener incentivos tributarios para quienes implementen y consoliden sistemas productivos de alimentos con carácter agroecológico, orgánico, biodinámico, permacultural o similares. Igualmente, para quienes pongan en práctica sistemas de buenas prácticas agrícolas y ganaderas. Asimismo, carácter especial deberían tener quienes en sus predios tengan áreas de conservación de bosques con vegetación nativa y que protejan fuentes de agua.

A este respecto el caso de Brasil con relación al Impuesto Territorial Rural (ITR) puede ser orientador sobre los aspectos para tener en cuenta, ya que este país viene implementando esta política desde finales del Siglo XIX (Neto, 1992). Su evolución ha mostrado, a juicio de algunos analistas, más fracasos que triunfos (Antunes, 2018). Si bien el ITR ha buscado desestimular la subutilización de la tierra, sus alcances han sido precarios y el peso del recaudo no supera el $1 \%$ del PIB debido a que hay un distanciamiento entre el contribuyente y los recaudadores (Neto, 1992), pero fundamentalmente a que la tasa de tributación está basada en la utilización del predio derivada de una relación entre el tamaño del mismo y su ocupación productiva. Los propietarios declaran una mayor ocupación productiva, por lo que la cuota impositiva disminuye y se evita que el predio sea sujeto de expropiación vía reforma agraria (Antunes, 
2018). Debido a que el ITR se basa en una autodeclaración por parte del propietario, su alcance cada vez es menor.

El caso brasilero lleva a una reflexión final que se relaciona con la credibilidad y fortaleza institucional, así como la importancia de la información que dicha institucionalidad posee para poder llevar a cabo sus funciones. Una institucionalidad centralizada, burocrática, que desconoce la realidad de las comunidades difícilmente puede emprender acciones que propendan por alcanzar la redistribución de la propiedad. La institucionalidad, por lo tanto, debe estar en estrecho contacto con la comunidad y actuar de la mano con ella para ganar la legitimidad que le permita obtener la información necesaria para alcanzar sus objetivos. Esto sin duda se alcanza al fortalecer los procesos de reforma agraria guiada por la comunidad.

\section{Conclusiones}

Este artículo buscaba analizar la distribución de las tierras rurales en Colombia durante las dos primeras décadas del Siglo XXI. En ese marco, se ha demostrado, con base en cifras oficiales del IGAC, que el problema histórico de la distribución de la propiedad rural en Colombia no se ha superado y que las discusiones a este respecto están cada vez más vigentes.

Un primer aspecto por destacar es que la distribución de la propiedad rural en Colombia es el reflejo de una situación estructural; un problema histórico de más de 500 años que se manifiesta en este caso en su concentración, pero que tiene otras manifestaciones en la sociedad como el mismo aislamiento de lo rural, por ejemplo.

Este problema estructural de distribución de la propiedad rural, analizado desde las cifras oficiales y en el coeficiente de Gini, demuestra que la política de reforma agraria ha beneficiado a los grandes propietarios en la medida en que sus condiciones continúan siendo beneficiosas.

Con relación a los resultados de la política de reforma agraria que toma cuerpo en la implementación de la RRI, la evidencia indica que los avances han sido muy precarios, casi inexistentes. Creándose una inmensa institucionalidad, en una serie de Agencias para la política agraria y el desarrollo rural, cuyo objetivo es lograr superar las inequidades en el sector rural; pero al parecer su enfoque y visión están replicando el modelo concebido y llevado a cabo desde inicios de la década de los años sesenta del siglo pasado, y que infortunadamente no logró resolver el problema de tierras ni de desarrollo rural en el país.

Sin embargo, la RRI no va a ser la solución a la concentración de la propiedad rural, debido a que, como se discutió a lo largo del artículo, esto es fruto de problemas estructurales que requieren otras formas de abordaje. En este sentido, ni la reforma agraria basada en el Estado, ni la basada en el mercado demostraron ser capaces de brindar solución a la concentración. Por tanto, una reforma agraria de base comunitaria podría ser la alternativa al problema, en la cual se entienda la tierra como un recurso de uso común, y se permita a las comunidades crear instituciones y formas alternativas de manejar el recurso.

Esta alternativa construida de abajo hacia arriba requiere que el Estado juegue un papel crucial para superar la desconfianza generalizada que tienen todos los actores involucrados en este problema. Una propuesta de esta naturaleza requiere unas bases comunitarias sólidas, pero a la vez una legislación que propenda a crear condiciones que permita conocer la realidad de la distribución de la propiedad rural basada en formas alternativas de abordar el problema, dando más peso a la idea de las Unidades Agrícolas Familiares.

Futuros trabajos de investigación se requieren para identificar precisamente a nivel territorial la problemática de la distribución. La visión nacional está bastante clara y naturalmente lo local debe ser muy similar; pero esa visión, más enfocada en lo que está sucediendo en ese nivel, 
debe ser especialmente analizado bajo la lógica de la UAF, para así lograr relacionarlo con la producción de alimentos, aspecto clave para consolidar procesos de reforma agraria guiada por la comunidad.

\section{Referencias}

Aliber, M., \& Cousins, B. (2013). Livelihoods after land reform in South Africa. Journal of Agrarian Change, 13(1), 140-165.

Antunes, T. G. (2018). O Imposto Territorial Rural (ITR) como ferramenta da gestão tributária. Tecnologia e Ambiente, 24, 215-232.

Bautista, R., Bazoberry, O., Gil, K., Chumacero, J., \& Soliz, L. (2017). Informe 2016. Acceso a la tierra y territorio en Sudamérica. La Paz: Instituto para el Desarrollo Rural de Sudamérica.

Berry, R. A. (2017). Reflections on injustice, inequality and land conflict in Colombia. Canadian Journal of Latin American and Caribbean Studies, 42(3), 277-297. https://doi.org/10.1080/ 08263663.2017.1378400.

Borras, S. M. (2003). Questioning market-led agrarian reform: experiences from Brazil, Colombia and South Africa. Journal of Agrarian Change, 3(3), 367-394. http://dx.doi.org/10.1111/14710366.00059

Borras, S. M., Hall, R., Scoones, I., White, B., \& Wolford, W. (2011). Towards a better understanding of global land grabbing: an editorial introduction. The Journal of Peasant Studies, 38(2), 209-216. http://dx.doi.org/10.1080/03066150.2011.559005

Botia-Carreño, W. H. (2019). Unidad Agrícola Familiar (UAF), instrumento de política pública agropecuaria en Colombia. Pensamiento y Acción, 27, 59-89.

Brittain, J. J. (2005). A theory of accelerating rural violence: lauchlin currie's role in underdeveloping Colombia. The Journal of Peasant Studies, 32(2), 335-360. http://dx.doi. org/10.1080/03066150500094535

Cárdenas, J. C. (2009). Dilemas De lo colectivo instituciones, pobreza y cooperación en el manejo local De los recursos De uso común. Bogotá: Ediciones Uniandes-Universidad de los. Andes (Salta).

Centro Nacional de Memoria Histórica. (2013). La política de reforma agraria y tierras en Colombia. Esbozo de una memoria institucional. Bogotá: Imprenta Nacional.

Colombia. Agencia Presidencial para la Acción Social y la Cooperación Internacional, Proyecto Protección de Tierras y Patrimonio de la Población. (2010). Unidades agrícolas familiares, tenencia y abandono forzado de tierras en Colombia. Bogotá: Equipo de Comunicaciones.

Comisión Económica para América Latina - CPAL. (2004). Una década de desarrollo social en América Latina, 1990-1999. Santiago de Chile: CEPAL.

Deinlnger, K., \& Binswanger, H. (1999). The evolution of the World Bank's land policy: principles, experience, and future challenges. The World Bank Research Observer, 14(2), 247-276. http://dx.doi.org/10.1093/wbro/14.2.247

Duarte, C., Duque, A. M., Cardoza, G., Castaño, A., Correa, J., Marmolejo, M., Montaño, M., Ochoa, M., Ramirez, C., \& Trujillo, D. (2019). La UAFy el ordenamiento territorial rural colombiano, 3 estudios de caso: Casanare, Cundinamarca y Bolívar. Cali: Sello Editorial Javeriano.

Echeñique, J. (2011). Dinámica del mercado de la tierra en América Latina y el Caribe: el caso de Chile. Santiago: FAO. 
Eguren, F. (2018). La agricultura en los paises andinos: Bolivia, Chile, Colombia, Ecuador, Peru y Venezuela: una revision de largo plazo 1961-2014. Agrarian, 186, 4-10.

Fajardo, D. (2002). Para sembrar la paz, hay que aflojar la tierra: Comunidades, tierras y territorios en la construcción de un país. Bogotá: Universidad Nacional de Colombia.

Favat, P. A., \& Gutiérrez, G. (2010). Crecimiento de la agricultura en Uruguay: exclusión social o integración económica en redes. Pampa: Revista Interuniversitaria de Estudios Territoriales, 6, 113-138.

Forero Rueda, S. (2020, Abril 13). Así va el Acuerdo de Paz: La deuda con la Reforma Rural Integral. El Espectador. Recuperado el 18 de agosto de 2020, de https://www.elespectador. com/colombia2020/pais/asi-va-el-acuerdo-de-paz-la-deuda-con-la-reforma-rural-integralarticulo-914280

Goebel, A. (2012). Gender and agrarian reforms. The Journal of Peasant Studies, 39(1), 204-206. http://dx.doi.org/10.1080/03066150.2012.656236

Gómez, C. J. L., Sánchez-Ayala, L., \& Vargas, G. A. (2015). Armed conflict, land grabs and primitive accumulation in Colombia: micro processes, macro trends and the puzzles in between. The Journal of Peasant Studies, 42(2), 255-274. http://dx.doi.org/10.1080/03066150.2014.990893

Grajales, J. (2015). Land grabbing, legal contention and institutional change in Colombia. The Journal of Peasant Studies, 42(3-4), 541-560. http://dx.doi.org/10.1080/03066150.2014.992883

Gras, C., \& Göbel, B. (2014). Agronegocio y desigualdades socioambientales: La soja en Argentina, Brasil y Uruguay. In B. Göbel, M. Góngora-Mera \& A. Ulloa (Orgs.), Desigualdades sociambientales en Amércia Latina (pp. 211-253). Bogotá: Editorial Universidad Nacional de Colombia.

Guereña, A., \& Burgos, S. O. (2016). Desterrados: Tierra, podery desigualdad en América Latina. Oxford: Oxfam.

Hardin, G. (1968). The tragedy of the commons. Science, 162(3859), 1243. http://dx.doi. org/10.1126/science.162.3859.1243

Hirschman, A. O. (2013). la tenencia de la tierra y la reforma agraria en Colombia. Empleo de las armas fiscales. Revista de Economia Institucional, 15, 351-360.

Hoffmann, R. (1979). Estimação da desigualdade dentro de estratos no cálculo do índice de Gini e da redundância. Pesquisa e Planejamento Econômico, 9(3), 719-738.

Hristov, J. (2005). Indigenous struggles for land and culture in Cauca, Colombia. The Journal of Peasant Studies, 32(1), 88-117. http://dx.doi.org/10.1080/0306615042000322402

Instituto Geográfico Agustín Codazzi. (2004). Estadísticas Catastrales. Distribución rural por rango de superficie. Bogotá: IGAC.

Instituto Geográfico Agustín Codazzi. (2010). Estadísticas Catastrales. Distribución rural por rango de superficie. Bogotá: IGAC.

Instituto Geográfico Agustín Codazzi. (2012). Atlas de la distribución de la propiedad rural en Colombia. Bogotá: IGAC.

Instituto Geográfico Agustín Codazzi. (2013). Estadísticas Catastrales. Distribución rural por rango de superficie. Bogotá: IGAC.

Instituto Geográfico Agustín Codazzi. (2014). Estadísticas Catastrales. Distribución rural por rango de superficie. Bogotá: IGAC.

Instituto Geográfico Agustín Codazzi. (2018). Estadísticas Catastrales. Distribución rural por rango de superficie. Bogotá: IGAC. 
Instituto Geográfico Agustín Codazzi. (2019). Estadísticas Catastrales. Distribución rural por rango de superficie. Bogotá: IGAC.

Karl, R. A. (2017). Century of the exile: Colombia's displacement and land restitution in historical perspective, 1940s-1960s. Canadian Journal of Latin American and Caribbean Studies, 42(3), 298-319. https://doi.org/10.1080/08263663.2017.1317951

Kay, C. (1998). Latin America's agrarian reform: lights and shadows. Land Reform, Land Settlement and Cooperatives, 2, 9-31.

Kay, C. (2002). Chile's neoliberal agrarian transformation and the peasantry. Journal of Agrarian Change, 2(4), 464-501. http://dx.doi.org/10.1111/1471-0366.00043

LeGrand, C. C., van Isschot, L., \& Riaño-Alcalá, P. (2017). Land, justice, and memory: Challenges for peace in Colombia. Canadian Journal of Latin American and Caribbean Studies, 42(3), 259-276. https://doi.org/10.1080/08263663.2017.1378381

Machado, A. (2009). La reforma rural, una deuda social y política. Bogotá: Universidad Nacional de Colombia. Facultad de Ciencias Económicas. Centro de Investigaciones para el Desarrollo CID.

Machado, A. (2013). La política de reforma agraria y tierras en Colombia. Esbozo de una memoria institucional. Bogotá: Centro Nacional de Memoria Histórica.

Machado, A., \& Vivas, J. (2009). Ensayos para la historia de la política de tierras en Colombia. De la colonia a la creación del Frente Nacional. Bogotá: Editorial Gente Nueva.

Neto, A. M. (1992). Imposto territorial rural (ITR): algumas considerações. Indicadores Econômicos FEE, 20(3), 185-199.

Nilsson, M., \& Taylor, L. K. (2017). Applying the security-development nexus on the ground: land restitution in Colombia. Conflict Security and Development, 17(1), 73-89. http://dx.doi.org /10.1080/14678802.2016.1231844

Ostrom, E. (2009). El gobierno de los bienes comunes: La evolución de las instituciones de acción colectiva (Números E14-295). Mexico: FCE;UNAM;CRIM;IIS.

Palau, M. (2019). Con la soja al cuello 2019. Informe sobre agronegocios en Paraguay. Asunción: BASE-IS. Recuperado el 18 de agosto de 2020, de http://www.baseis.org.py/wp-content/ uploads/2019/12/SAC19web_compressed.pdf

Petras, J., \& Veltmeyer, H. (2002). The peasantry and the state in Latin America: a troubled past, an uncertain future. The Journal of Peasant Studies, 29(3-4), 41-82. http://dx.doi.org/10.1 080/03066150412331311029

Quijano-Mejia, C. M., \& Linares-García, J. (2017). Zonas de reserva campesina: territorialidades en disputa. El caso del Valle del río Cimitarra. Prospectiva. Revista de Trabajo Social e Intervención Social, (24), 225-251.

Quintero, J. C. (1988). ¿ Qué pasó con la tierra prometida. Bogotá: CINEP.

Reboratti, C. (2010). Un mar de soja: la nueva agricultura en Argentina y sus consecuencias. Revista de Geografía Norte Grande, 45, 63-76. http://dx.doi.org/10.4067/S0718-34022010000100005

Sikor, T., \& Müller, D. (2009). The limits of state-led land reform: an introduction. The Limits of State-Led Land Reform, 37(8), 1307-1316. http://dx.doi.org/10.1016/j.worlddev.2008.08.010

Souza, S. S., \& Silva, E. A. (2011). Regional planning in the land reform literature: a gap to be bridged. Regional Studies, 45(6), 857-868. http://dx.doi.org/10.1080/00343400903496386

Thomson, F. (2011). The agrarian question and violence in Colombia: conflict and development. Journal of Agrarian Change, 11(3), 321-356. http://dx.doi.org/10.1111/j.1471-0366.2011.00314.x 
Uruguay. Ministerio de Ganadería, Agricultura y Pesca. (2015). Anuario estadístico Agropecuario 2014. Bogotá: Diea. Recuperado el 18 de agosto de 2020, de http://www.mgap.gub.uy/ Dieaanterior/Anuario2014/Diea-Anuario\%202014-Digital01.pdf

Von Benda-Beckmann, F., Von Benda-Beckmann, K., \& Wiber, M. (2006). The properties of property. Changing Properties of Property, 40, 1-39.

Recibido: Agosto 18, 2020

Aprobado: Mayo 20, 2021

JEL Classification: Q1, Q15. 